\title{
Projections of increased and decreased dengue incidence under climate change
}

\author{
C. R. WILLIAMS ${ }^{1,2}$, G. MINCHAM ${ }^{1}$, H. FADDY ${ }^{3}$, E. VIENNET ${ }^{2}$, \\ S. A. RITCHIE ${ }^{4}$ AND D. HARLEY ${ }^{2}$ \\ ${ }^{1}$ Centre for Population Health Research, University of South Australia, Adelaide, Australia \\ ${ }^{2}$ National Centre for Epidemiology and Population Health, Australian National University, Canberra, Australia \\ ${ }^{3}$ Australian Red Cross Blood Service, Brisbane, Queensland, Australia \\ ${ }^{4}$ School of Public Health, Tropical Medicine and Rehabilitation Sciences, James Cook University, Cairns, \\ Queensland, Australia
}

Received 8 February 2016; Final revision 1 June 2016; Accepted 1 July 2016; first published online 26 July 2016

\section{SUMMARY}

Dengue is the world's most prevalent mosquito-borne disease, with more than 200 million people each year becoming infected. We used a mechanistic virus transmission model to determine whether climate warming would change dengue transmission in Australia. Using two climate models each with two carbon emission scenarios, we calculated future dengue epidemic potential for the period 2046-2064. Using the ECHAM5 model, decreased dengue transmission was predicted under the A2 carbon emission scenario, whereas some increases are likely under the B1 scenario. Dengue epidemic potential may decrease under climate warming due to mosquito breeding sites becoming drier and mosquito survivorship declining. These results contradict most previous studies that use correlative models to show increased dengue transmission under climate warming. Dengue epidemiology is determined by a complex interplay between climatic, human host, and pathogen factors. It is therefore naive to assume a simple relationship between climate and incidence, and incorrect to state that climate warming will uniformly increase dengue transmission, although in general the health impacts of climate change will be negative.

Key words: Arboviruses, climate (impact of), dengue fever, infectious disease epidemiology.

\section{INTRODUCTION}

Dengue is the world's most common mosquito-borne viral infection of humans, with an estimated 284-528 million infections occurring annually [1]. Global incidence of dengue has increased in recent decades, with outbreaks of ever-increasing size being recorded in urban centres [2]. In Australia, dengue is an epidemic disease, with importations via viraemic travellers

\footnotetext{
* Author for correspondence: Dr C. R. Williams, Centre for Population Health Research, University of South Australia, Adelaide, Australia.

(Email: craig.williams@unisa.edu.au)
}

causing autochthonous transmission in the wet tropics of the country's northeast. Outbreaks of several hundred cases are recorded regularly in this region, and the size of locally transmitted outbreaks is steadily increasing [3], as is the number of imported infections, most commonly from Southeast Asia and Papua New Guinea [4, 5].

Mosquitoes require appropriate climatic conditions, and thus changes to the Earth's climate that have occurred and are predicted for the future [6] will impact the transmission of diseases they transmit. A number of models linking meteorological variables, climate and dengue transmission have been developed. 
Some are empirical models, whereby dengue transmission output variables are related statistically to meteorological data inputs (e.g. [7, 8]), whereas others make use of process-based (i.e. mechanistic) models, some of which include vector life-table calculations [9].

Predictions of increased geographical range for dengue and consequent increased population risk have been made (e.g. [10, 11]). Jetten \& Focks [12] used a version of the vectorial capacity equation to calculate the relative increase in dengue transmission intensity caused by a $2-4{ }^{\circ} \mathrm{C}$ increase in global temperature, demonstrating increased intensity and geographical range. Patz and others [10] used a similar approach to demonstrate increased dengue transmission potential with a $1.16^{\circ} \mathrm{C}$ average global temperature increase, while Hales et al. [11] created a logistic regression model to describe the current global dengue transmission range in terms of vapour pressure (a measure of atmospheric moisture) and predicted extension of the dengue transmission range. More recently, Liu-Helmersson and others [13] used expressions of vectorial capacity to calculate dengue epidemic potential (the propensity of mosquitoes to transmit dengue). Importantly, they demonstrated the strong temperature dependence of dengue epidemic potential, and also showed that diurnal temperature variation can have positive and negative impacts on dengue transmission.

Projections based on statistical models trained on existing dengue transmission ranges do not necessarily reflect the current climatological limits to dengue transmission. In Australia, dengue transmission has historically occurred over much of the eastern and western seaboards, and only retracted into its current North Queensland range in the past 50 years [14]. This suggests that the current climate is suitable for dengue transmission across a much greater range than currently occurs.

More recently, Åstrom and others [15] proposed an empirical model of dengue risk under future climate that included socioeconomic factors as predictive variables. A decrease in dengue transmission was predicted for some regions if socioeconomic and climate factors change as predicted. This work modelled historical dengue ranges and then took into account increases in financial prosperity; a negative influence in dengue transmission owing primarily to decreased mosquito-human contact rates.

Mechanistic, process based models explicitly describe the relationships between the local environment, disease vectors (e.g. mosquitoes), the pathogen and humans. Such models have been recommended for a more holistic study of dengue-climate relationships [12]. The Dengue Simulation Model (DENSiM) has been used to model dengue transmission in endemic and epidemic settings [16]. In a study of dengue outbreaks in North Queensland, DENSiM was parameterized for Australian conditions and used to demonstrate the role of weather as a determinant of transmission [9]. DENSiM was also used to test the impact of changing virus importation rate, and climate change, on dengue transmission in Malaysia [17]. The entomological component of DENSiM was recently used to determine likely changes to vector bionomics under climate warming [18]. Having such a validated, mechanistic dengue simulation model at our disposal presents an opportunity to test dengue receptivity of different regions from first principles. That is, to test the suitability of local climate (and in turn climate change) without relying on previous dengue geographical range data which have inherent biases in reporting.

Using the parameterized DENSiM model for Australia [9], in this study we characterized the climate-determined dengue receptivity of some key Australian urban centres. Then, using climate change projections for the mid-21st century, we determined whether dengue receptivity would change. Our conclusions have important public health implications for Australia, as well as more general significance for climate and health research.

\section{METHODS}

\section{DENSiM and dengue receptivity measures}

DENSiM [16], parameterized for Australian conditions [9], was used to assess dengue receptivity in Queensland for current and future climates. This parameterization involved the training and validation of both entomological (Supplementary Table S1) and human population parameters, such as age-specific birth and death rates and population densities [9]. In brief, DENSiM (v. 3.27, University of California, USA) is a mechanistic computer simulation of daily dengue transmission in a human population. Humans may become infected and in turn immune, to dengue virus, which can be introduced via viraemic humans or infected mosquitoes at rates and times specified by the user. DENSiM is supported by entomological inputs from the CIMSiM model, which is driven by daily meteorological observations [9]. Dengue receptivity is the ease with which dengue can be transmitted 
Table 1. Dengue receptivity measure descriptions and method of calculation

\begin{tabular}{llll}
\hline \hline & Description & How calculated & Reference \\
\hline $\begin{array}{l}\text { Outbreak } \\
\text { probability }\end{array}$ & $\begin{array}{c}\text { Probability of a new outbreak starting as } \\
\text { a result of a single viraemic introduction }\end{array}$ & $\begin{array}{c}\text { No. of local transmission events/ no. of } \\
\text { viraemic introductions in DENSiM }\end{array}$ & Present study \\
$\begin{array}{c}\text { Threshold mosquito } \\
\text { density }(m)\end{array}$ & $\begin{array}{c}\text { Average mosquitoes per person required } \\
\text { to infect one additional person }\end{array}$ & $\begin{array}{c}\text { Rearranged vectorial capacity (VC) } \\
\text { expression to solve for } m \text { when VC }=1\end{array}$ & {$[15,18]$} \\
& $\begin{array}{l}\text { Reciprocal of threshold density (above), } \\
\text { a measure of dengue receptivity }\end{array}$ & & {$[15]$} \\
\hline \hline
\end{tabular}

in an environment should the virus be present. Here we characterized dengue receptivity in terms of outbreak probability and epidemic potential (Table 1). The latter is the reciprocal of threshold mosquito density, the number of mosquitoes required to sustain local transmission. While we use the term 'epidemic potential' coined by others $[12,13]$ we interpret meaning to be relating to local transmission of new cases, rather than satisfying local criteria for epidemic definition. The fewer mosquitoes that are required to sustain transmission, the greater the probability of local transmission (epidemic potential).

\section{Modelling of dengue receptivity in current and future climates}

We calculated epidemic potential (Table 1) in the current climate (1990-2011) for peak transmission months in Australia (December, January, February). Calculations were also made for July, the month with the lowest average incidence.

Calculations were made for four urban centres in Queensland (Fig. 1). Cairns (population 224 000) and Townsville (218000) are within the current dengue transmission range, with transmission recorded in both cities in most years [5, 19]. Aedes aegypti is present in Rockhampton (60000) but there have been no incident dengue cases in recent decades [14]. Neither Ae. aegypti nor incident dengue currently occur in Brisbane (2 066 000) (last recorded in 1956) [14].

\section{Threshold mosquito density (m) calculations made using DENSiM}

Simulations of mosquito populations were made for each location for the period 1990-2011 (21 years) using the CIMSiM sub-model within DENSiM [20]. For December, January, February, and July, threshold mosquito density was calculated using the method of Jetten \& Focks [12], whereby the vectorial capacity equation [21] was rearranged so that mosquito abundance $(m)$ could be calculated when the attack rate (vectorial capacity) was set to 1 (sustaining transmission). To enable calculation of $m$ for each month, DENSiM default settings for probability of transmission from each viraemic mosquito with access to each human in the simulation $(0 \cdot 9)$ and human to mosquito $(0.75)$ were used [16]. A daily adult mosquito survivorship of 0.83 [20] was used as a constant. From DENSiM simulations, average monthly values of bites per person per day, and extrinsic virus incubation period, both of which vary with weather conditions and time, were obtained from simulation outputs. The following equation [12] was used to calculate threshold mosquito density:

$m=\frac{-\ln (P)}{b c a^{2} P^{n}}$,

where $m=$ the threshold density required for dengue transmission, $P$ is the daily survival probability for an individual mosquito, $b$ is the probability that an infectious mosquito transmits dengue while biting a susceptible human, $c$ is the probability that a mosquito acquires dengue infection while biting a viraemic human, $a$ is the number of bites per person per day, and $n$ is the duration in days of the extrinsic incubation period.

Source information for these parameters is described in Table 2.

\section{Process-based modelling using DENSiM}

\section{Meteorological data and climate change scenarios}

Current climate in this study comprised a 21-year period, from 1990 to 2011, with daily meteorological observations (maximum, minimum, and average temperature, rainfall, relative humidity, saturation deficit). Daily meteorological values for future climate were obtained for the period 2046-2064 from the Australian Bureau of Meteorology. To derive 


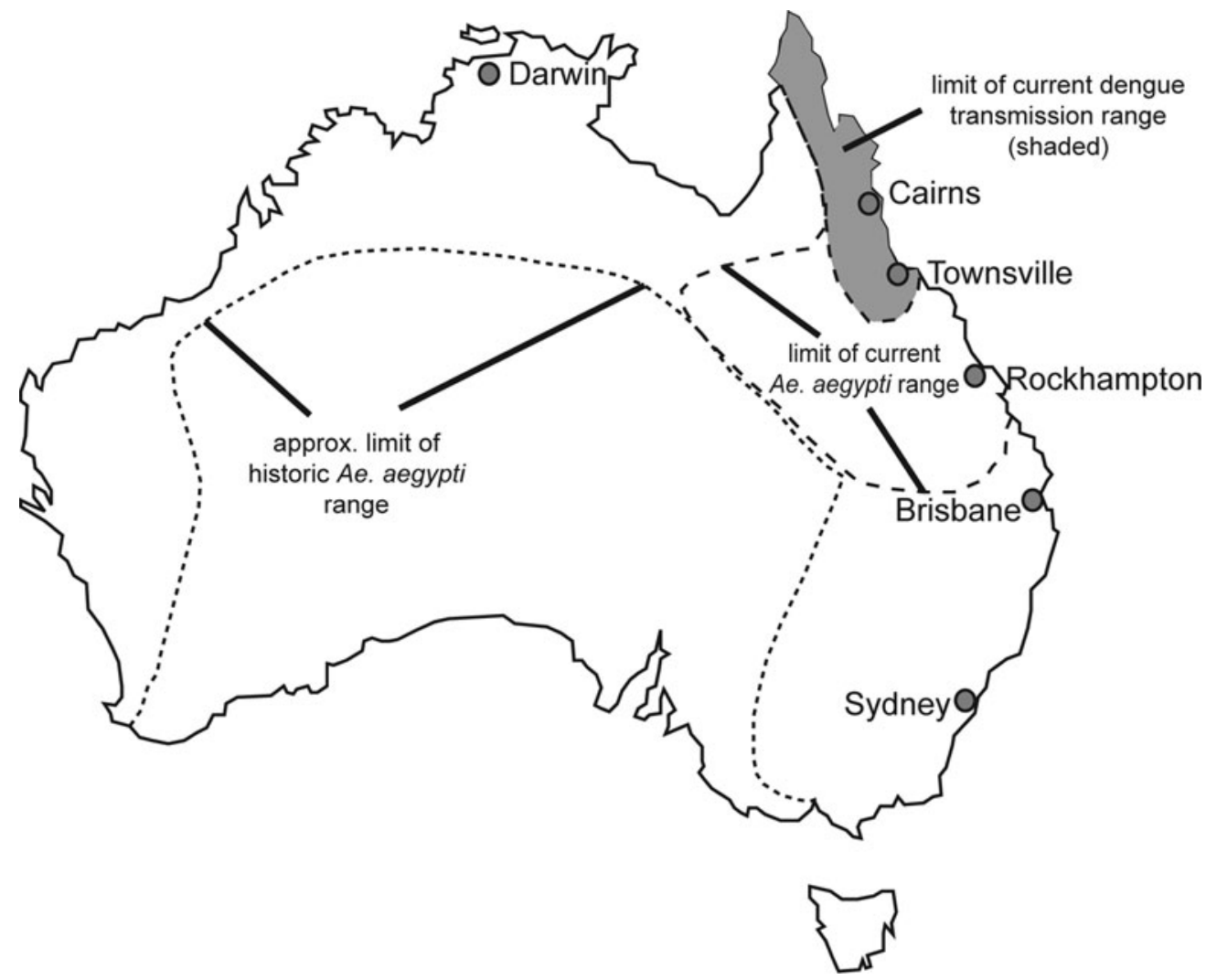

Fig. 1. Map of current dengue transmission and vector range in Australia and location of cities tested for dengue receptivity in this study (modelling not performed for Darwin and Sydney).

Table 2. Parameters of the vectorial capacity equation and their sources

\begin{tabular}{llll}
\hline \hline Description & How derived & Reference \\
\hline$P$ Daily survival probability for & $\begin{array}{c}\text { Constant value of 0.83 used in DENSiM model } \\
\text { based on field observations }\end{array}$ & {$[19]$} \\
$b \begin{array}{l}\text { Probability infectious mosquito } \\
\text { biting human }\end{array}$ & Constant value used in DENSiM model & {$[9]$} \\
$c \quad \begin{array}{l}\text { Probability human acquires } \\
\text { infection from mosquito }\end{array}$ & Constant used in DENSiM model & {$[9]$} \\
$a$ & Bites per person per day & From DENSiM simulations. Host-seeking females \\
$n$ & per ha/human density, based on local climate & {$[25]$} \\
$n$ & Extrinsic incubation period & From DENSiM simulations, based on local climate & {$[25]$} \\
\hline \hline
\end{tabular}

plausible daily values from long-term projection data, a statistical downscaling technique was used [22]. In brief, this involved creation of a downscaling model, which takes large-scale (in both time and space) regional climate change predictions, and converts them to daily timescale and single-point spatial predictions. The models are tested for accuracy using historical meteorological observations [22]. Values for current and future climate were obtained from the Australian Bureau of Meteorology (B. Timbal, Y. Wang, and A. Evans, Melbourne Office).
Future climate was predicted by the Max Planck Institute's ECHAM5 global circulation model, based on its reported skill for climate prediction in Australia [23]. Two SRES carbon emission scenarios were investigated. The $\mathrm{B} 1$ future climate scenario incorporates rapid economic growth, a global population rise to 9 billion in 2050, changes towards a service and information economy, and some reductions in material intensity. The A2 scenario was also used, with slower transition to renewable energy sources and higher carbon emissions [6]. In general, temperatures are expected 
to increase in all cities tested here under both emission scenarios, although the magnitude of temperature change varies with carbon scenario. The outlook for rainfall is mixed, with some decreases expected in Rockhampton and Brisbane, and increases predicted in Cairns and Townsville (see Supplementary material).

\section{Simulation locations}

Simulations were performed for a fixed human population size in each location. The cities are not demographically homogeneous, but rather are composed of clusters of suburbs. For this reason, we decided to test dengue receptivity in groups of 10000 people for the first year of each simulation. Demographic data (birth and death rates, age group size) were obtained from the Australian Bureau of Statistics. Population sizes in each location increased from the initial 10000 according to the birth and death rates programmed into DENSiM.

Receptivity was characterized by outbreak probability and epidemic potential (reciprocal of the threshold density of mosquitoes required for transmission; Table 1). Receptivity was evaluated for December, January, February (northern Australian wet season when dengue incidence is highest) and July (dry season, low dengue incidence). For each simulation run, a single viraemic introduction was made for the selected month and year, making each year a replicate for each climate scenario (current and future). Thus, for each emission scenario and climate scenario (current and future climate), and each of the four cities, virus was introduced separately in four different months (December, January, February, July). Each simulation length was 21 years, with each year acting as a replicate. This gave a total of 48 simulation variants, each being 21 years long. These in turn were replicated 10 times each to calculate mean values (DENSiM has stochastic functions in-built, with variations in food delivery to mosquito larvae). Thus, a total of 480 separate simulations were performed, with data extracted to calculate the parameters described in Table 1.

\section{RESULTS}

\section{Probability of dengue outbreaks and epidemic potential}

\section{Current climate}

Dengue transmission is possible in the current climate in all study centres, with Cairns most suitable and Brisbane least. Probability of local dengue transmission in the current climate following a single viraemic introduction in December, January or February was $100 \%$ for Cairns. For other cities the transmission probability varied depending on month. Transmission probability ranged from $5 \%$ to $57 \%$ for Townsville (peaking in February) (Figs 2 and 3). Probability of transmission under current climate was high for Rockhampton (10-86\%, peaking in January), and was low for Brisbane (10-33\%, also peaking in February, with no transmission predicted in December). Epidemic potential was highest for Cairns and lowest for Brisbane (Fig. 4).

\section{Future climate}

In the future, location and emission scenario will determine whether epidemic potential increases or decreases (Fig. 4). Epidemic potential is predicted to increase for Townsville and Rockhampton under the B1 emission scenario. A predicted decrease for epidemic potential for all cities is made under the A2 emission scenario using MPI ECHAM 5 (Fig. 4).

In a future climate, outbreak probability in Cairns is predicted to be unchanged from $100 \%$ under the B1 scenario, but may decrease slightly under the A2 scenario (Figs 3 and 4). The B1 scenario also produces predictions of increased dengue outbreak probability for Rockhampton, Townsville, and Brisbane, whereas under the A2 scenario is predictive of decreased outbreak probability.

\section{DISCUSSION}

Most studies of dengue under climate change for Australia (and globally) predict increases in incidence and range. Contrary to earlier studies we predict that based on climate changes alone, dengue transmission in Australia may remain stable, increase, or even decrease in the future. Our modelling incorporates not only mosquito virus carriage and ecology, but also human serology, and biting rates. This work does not rely on previous training of statistical models against the current dengue transmission range, but rather uses a mechanistic approach to calculate both mosquito abundance and infectivity, together with human infection.

Predicted decreases in dengue receptivity are primarily due to changes in vector abundance, size and survivorship. In previous studies we demonstrated that climate change will likely reduce $A e$ aegypti abundance in Queensland under the A2 scenario, due to greater evaporation and temperatures reducing 

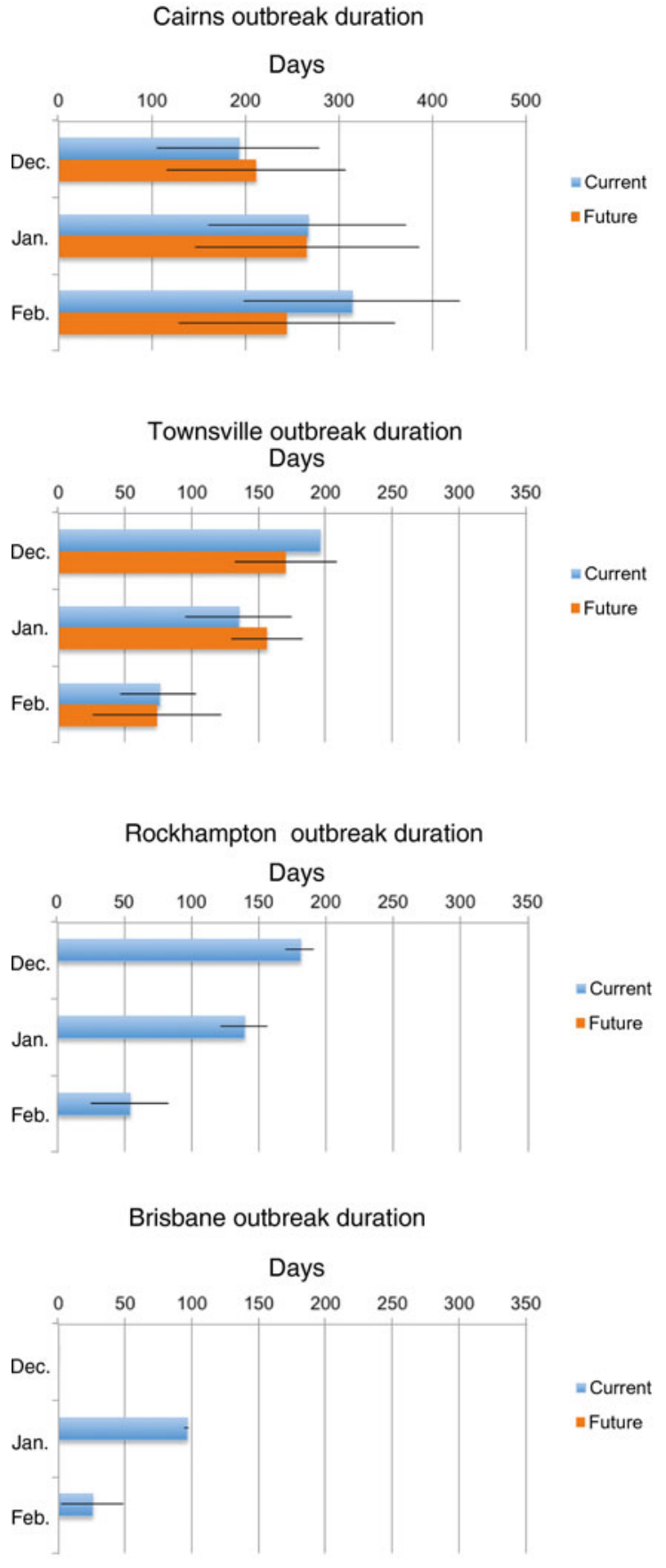
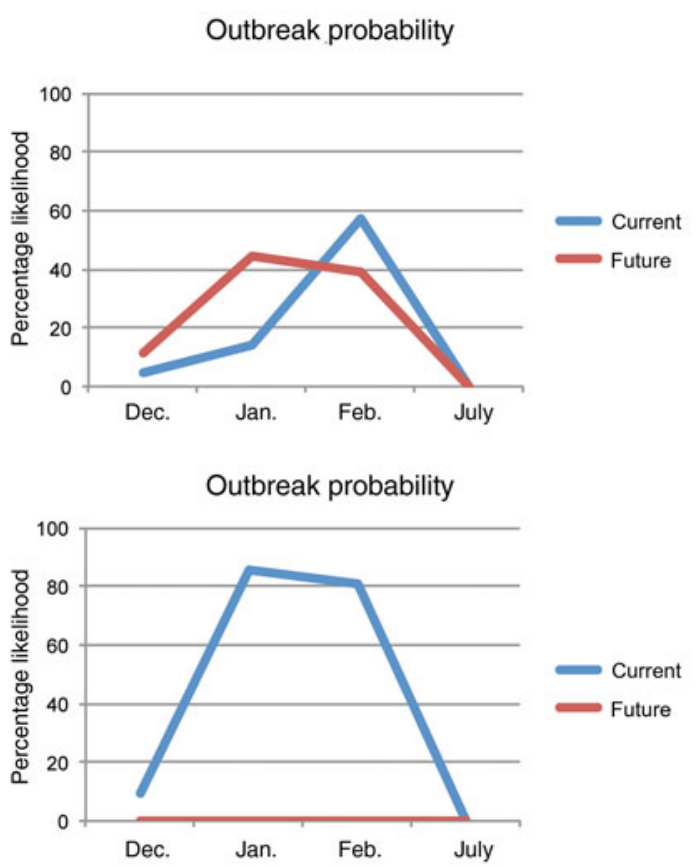

Outbreak probability

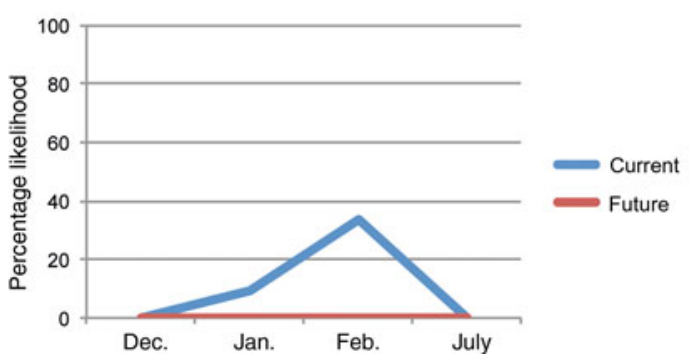

Fig. 2. Dengue receptivity in both current and future climate (A2 scenario, ECHAM5 model) for four Australian cities.

breeding site productivity, and temperatures exceeding the thermal optima for the species more often than in the current climate [18]. A prediction for further decreases in dengue vector abundance in southern Queensland is consistent with historical observations of the retraction of dengue from this region since the mid-20th century [14]. Future dengue receptivity calculations in this study for the A2 scenario all decrease, a finding consistent with predicted future mosquito bionomics. Conversely, some increases in dengue receptivity under the B1 scenario were predicted for Townsville and Rockhampton, a finding consistent with the increased mosquito abundance previously predicted for the B1 scenario [14].

These results also demonstrate that the extent of climate change that occurs in the future will partly determine changes to dengue transmission. However, it would seem that higher carbon emissions may result 

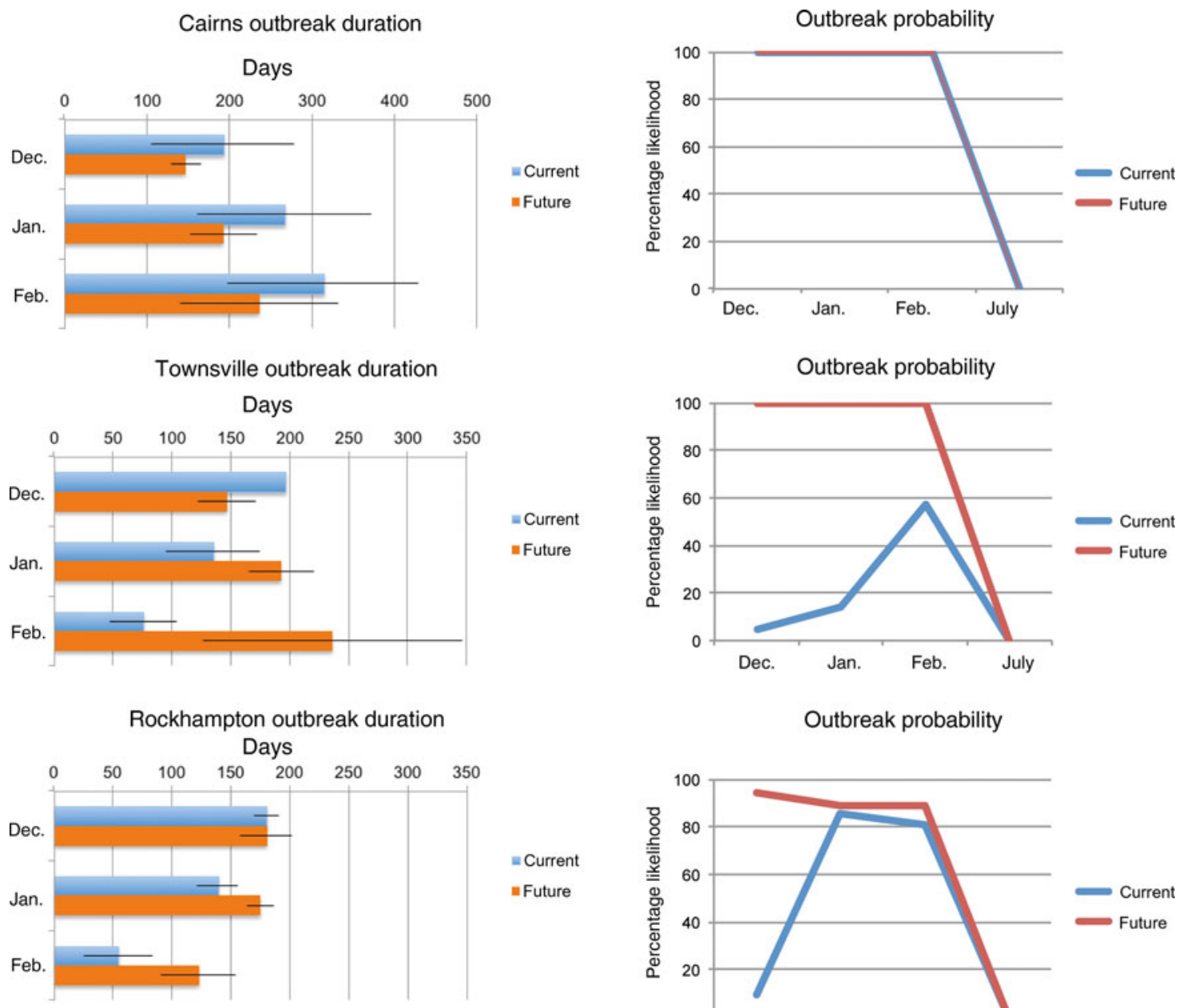

Outbreak probability
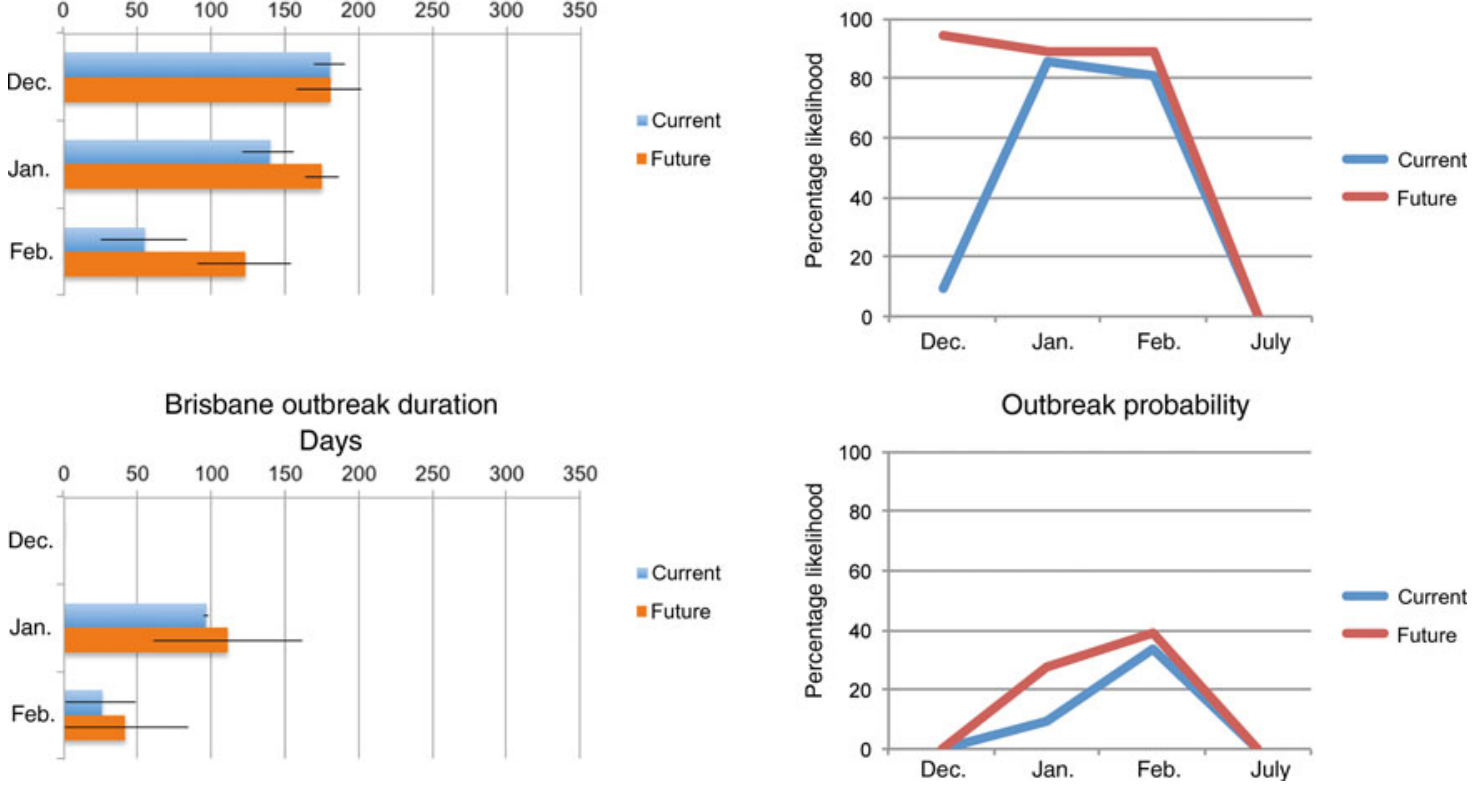

Fig. 3. Dengue receptivity in both current and future climate (B1 scenario, ECHAM5 model) for four Australian cities.

in less dengue, whereas milder climate warming due to slower increase in carbon emissions may actually lead to more dengue. Our results are consistent with recent findings that the relationship between climate and dengue is likely to be context-dependent and that decreases in dengue under climate change are to be expected in some locations [24]. Furthermore, previous work [13] has shown that diurnal temperature variation may either increase or decrease dengue epidemic potential, depending on the extent of variation. While our work does not consider diurnal temperature variation, we incorporate other complex vector ecology variables (through the DENSiM model) that also indicate the potential for dengue transmission to decrease in a warming climate.

There are factors other than climate that influence dengue transmission, as evidenced by past dengue activity patterns in Australia [5]. While dengue 


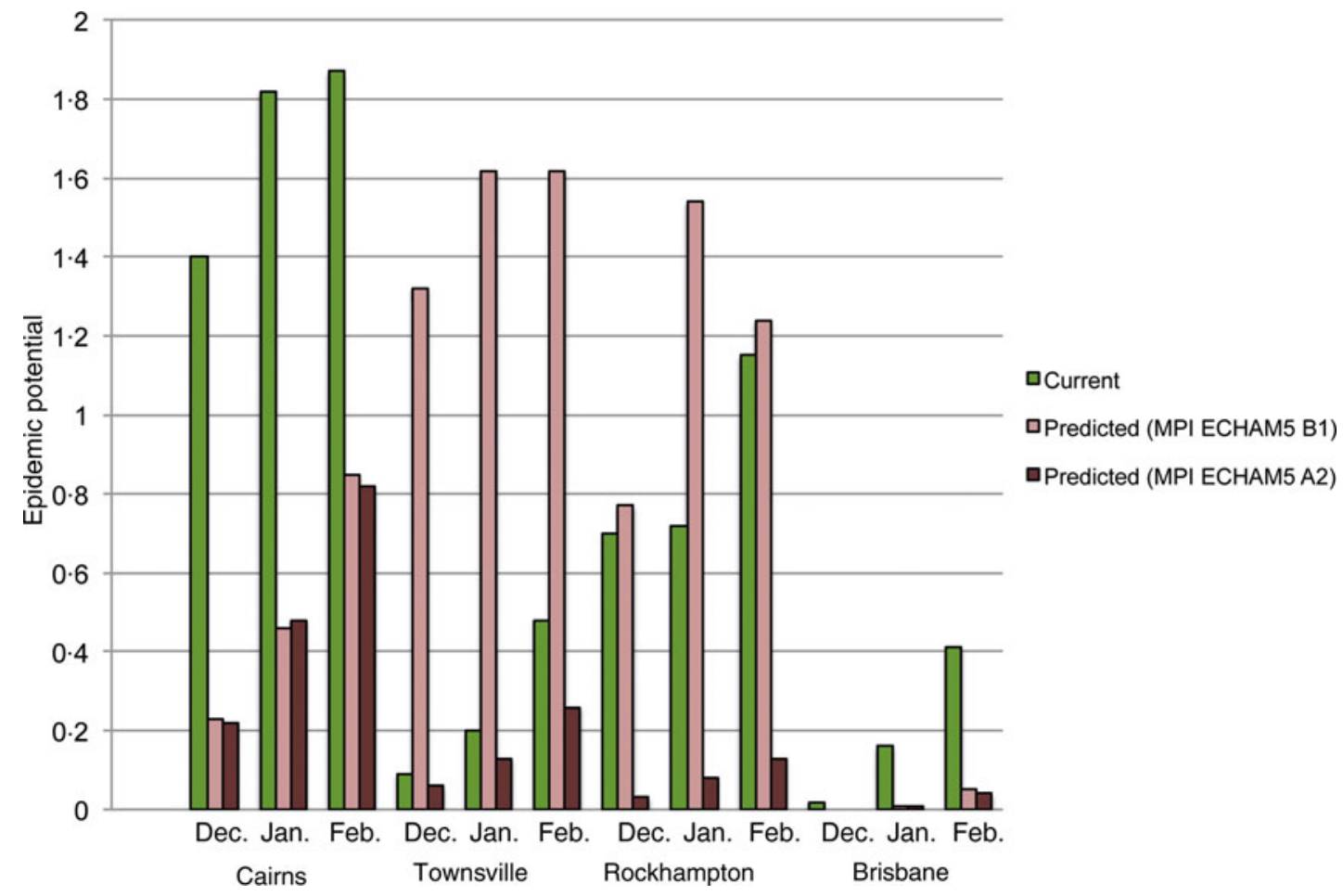

Fig. 4. Simulated dengue epidemic potential for Queensland cities under climate change for 2046-2064 compared with current climate (1991-2011).

transmission in Australia is currently restricted to northern Queensland, the principal vector, Ae. aegypti, occurs further south. Dengue transmission previously occurred over a much greater area in Australia. Indeed, our results here indicate that the current climate is suitable for some dengue transmission in all urban centres tested, including Brisbane where dengue has not been transmitted since the 1950s. Previous studies $[14,25]$ have described factors responsible for the geographical contraction of dengue in Australia, which occurred during a period of climate warming from the mid-20th century. Possible causes of dengue range retraction include improved water reticulation, insecticide usage, and the decline in water storage after steam rail transport was phased out in the mid-20th century.

While we have found that climate warming is unlikely to cause increases in dengue epidemic potential in areas where transmission has historically occurred, it remains vital that efforts to mitigate and adapt to the effects of climate change continue. Climate is not the only determinant of mosquito-borne disease transmission, with increases in human population size, travel, and virus mutation posing real threats to future human health. Thus, strategies to mitigate the impact of dengue in the future (e.g. enhanced surveillance and case management, vaccine development, use of symbiont Wolbachia bacteria to control vectors [26] remain vital, regardless of whether climate warming is responsible for increasing dengue risk. It is also probable that other climate impacts on health including on food security, migration, and conflict, will be far more significant than any changes in dengue incidence [27].

There are some obvious limitations to our study. One of which is the choice of simulation model used here. While DENSiM offers highly detailed, locationspecific simulation, replicate model runs and data extraction can be cumbersome. This naturally limited our ability to test dengue receptivity for all but four months of the year and for four locations. Future approaches should perhaps consider simpler ways to estimate mosquito abundance that permit modelling epidemic potential on broader spatial and temporal scales.

Our modelling suggests that a simplistic extrapolation based on current climate and transmission patterns may neglect factors important in transmission dynamics, such as vector bionomics, and hence lead to wrong conclusions and the misallocation of resources. For instance, a prediction of disease increase in a highly populous region may result in 
significant resource allocation for the formulation of action plans, investment in surveillance and vector control. If predictions are inaccurate, this may result in unnecessary funding and may shake faith in other disease model predictions.

It is important to maintain capacity to respond to dengue outbreaks in Australia. Moreover, as dengue can be transmitted through blood transfusion [28], leading to restrictions on blood donations during local outbreaks in Australia [29] changes to dengue transmission in the future may impact on blood collections in these cities and therefore impact on the location of future donation collection facilities.

Our study represents a methodological advance in that current climate-dengue relations are not assumed to hold under future climate and also via the incorporation of sophisticated biologically based understanding of vector biology. We acknowledge that unanticipated changes in demography, travel patterns, global dengue epidemiology, and vector and virus biology may significantly impact predictions for dengue. But the modelling results we have presented suggest caution is appropriate when extrapolating future dengue incidence under climate change based on current climate-dengue relations.

\section{SUPPLEMENTARY MATERIAL}

For supplementary material accompanying this paper visit http://dx.doi.org/10.1017/S095026881600162X.

\section{ACKNOWLEDGEMENTS}

Tom Scott and Andy Garcia (University of California) provided CIMSiM version 3.27 and much technical advice and support for its use. Alex Evans, Bertrand Timbal and Yan Wang at the Bureau of Meteorology (Melbourne) were very helpful in obtaining statistically downscaled data for future climate. Melanie Bannister-Tyrrell was pivotal in the continued application of CIMSiM in Australia. Anonymous reviewers provided comments that significantly improved the manuscript.

This project was funded by the Commonwealth Department for Climate Change, via the NH\&MRC (project 1003 371).

\section{DECLARATION OF INTEREST}

None.

\section{REFERENCES}

1. Bhatt S, et al. The global distribution and burden of dengue. Nature 2013; 496: 504-507.

2. World Health Organization. Impact of dengue (http:// www.who.int/csr/disease/dengue/impact/en/). Accessed 29 August 2013.

3. Vazquez-Prokopec GM, et al. Quantifying the spatial dimension of dengue virus epidemic spread within a tropical urban environment. PLoS Neglected Tropical Diseases 2010; 4(12): e920.

4. Knope K, Giele C. Increasing notifications of dengue in Australia related to overseas travel, 1991 to 2012. Communicable Diseases Intelligence 2013; 37: E55-59.

5. Viennet E, et al. Epidemiology of dengue in a highincome country: a case study in Queensland, Australia. Parasites and Vectors 2014; 7: 379.

6. IPCC. Climate Change 2014: Synthesis Report. Contribution of Working Groups I, II and III to the Fifth Assessment Report of the Intergovernmental Panel on Climate Change (Core Writing Team, R. K. Pachauri and L. A. Meyer, eds). IPCC, Geneva, Switzerland, 2014, 151 pp.

7. Bi P, et al. Climate variability and the dengue outbreak in Townsville, Queensland, 1992-93. Environmental Health 2001; 1: 54.

8. Descloux E, et al. Climate-based models for understanding and forecasting dengue epidemics. PLoS Neglected Tropical Diseases 2012; 6: e1470.

9. Bannister-Tyrrell M, et al. Weather-driven variation in dengue activity in Australia examined using a processbased modeling approach. American Journal of Tropical Medicine and Hygiene 2013; 88: 65-72.

10. Patz JA, et al. Impact of regional climate change on human health. Nature 2005; 438, 310-317.

11. Hales $\mathbf{S}$, et al. Potential effect of population and climate changes on global distribution of dengue fever: an empirical model. Lancet 2002; 360: 830-834.

12. Jetten TH, Focks DA. Potential changes in the distribution of dengue transmission under climate warming. American Journal of Tropical Medicine and Hygiene 1997; 57: 285-297.

13. Liu-Helmersson $\mathbf{J}$, et al. Vectorial capacity of Aedes aegypti: effects of temperature and implications for global dengue epidemic potential. PLoS ONE 2014; 9: e89783.

14. Russell RC, et al. Dengue and climate change in Australia: predictions for the future should incorporate knowledge from the past. Medical Journal of Australia 2009; 190: 265-268.

15. Åström C, et al. Potential distribution of dengue fever under scenarios of climate change and economic development. Ecohealth 2012; 9: 448-454.

16. Focks DA, et al. A simulation model of the epidemiology of urban dengue fever: literature analysis, model development, preliminary validation, and samples of simulation results. American Journal of Tropical Medicine and Hygiene 1995; 53: 489-506.

17. Williams CR, et al. Testing the impact of virus importation rates and future climate change on dengue activity 
in Malaysia using a mechanistic entomology and disease model. Epidemiology and Infection 2015; 143: 2856-2864.

18. Williams CR, et al. Bionomic response of Aedes aegypti to two future climate change scenarios in Far North Queensland, Australia: implications for dengue outbreaks. Parasites and Vectors 2014; 7: 447.

19. Hanna J, Ritchie SA. Outbreaks of dengue in North Queensland 1990-2008. Communicable Diseases Intelligence 2009; 33: 32-33.

20. Williams CR, et al. Rapid estimation of Aedes aegypti population size using simulation modeling, with a novel approach to calibration and field validation. Journal of Medical Entomology 2008; 45: 1173-1179.

21. Macdonald G. The Epidemiology and Control of Malaria. London: Oxford University Press, 1957.

22. Timbal B, Fernandez E, Li Z. Generalization of a statistical downscaling model to provide local climate change projections for Australia. Environmental Modelling and Software 2009; 24: 341-358.

23. Perkins SE, Pitman AJ, Sisson SA. Systematic differences in future 20 year temperature extremes in AR4 model projections over Australia as a function of model skill. International Journal of Climatology 2013; 33: $1153-1167$.

24. Morin CW, Comrie AC, Ernst KC. Climate and dengue transmission: evidence and implications. Environmental Health Perspectives 2013; 121: 1264-1272.

25. Williams CR, et al. The extinction of dengue through natural vulnerability of its vectors. PLoS Neglected Tropical Diseases 2010; 4: e922.

26. Hoffmann AA, et al. Successful establishment of Wolbachia in Aedes populations to suppress dengue transmission. Nature 2011; 476: 454-457.

27. McMichael AJ, Woodruff RE, Hales S. Climate change and human health: present and future risks. Lancet 2006; 367: 859-869.

28. Levi JE, et al. Real-time symptomatic case of transfusion-transmitted dengue. Transfusion 2015; 55: 961-964.

29. Faddy HM, et al. Implications of dengue outbreaks for blood supply, Australia. Emerging Infectious Diseases 2013; 19: 787. 